\title{
‡USGS
}

science for a changing world

Geochemical Results from Stream-Water and StreamSediment Samples Collected in Colorado and New Mexico

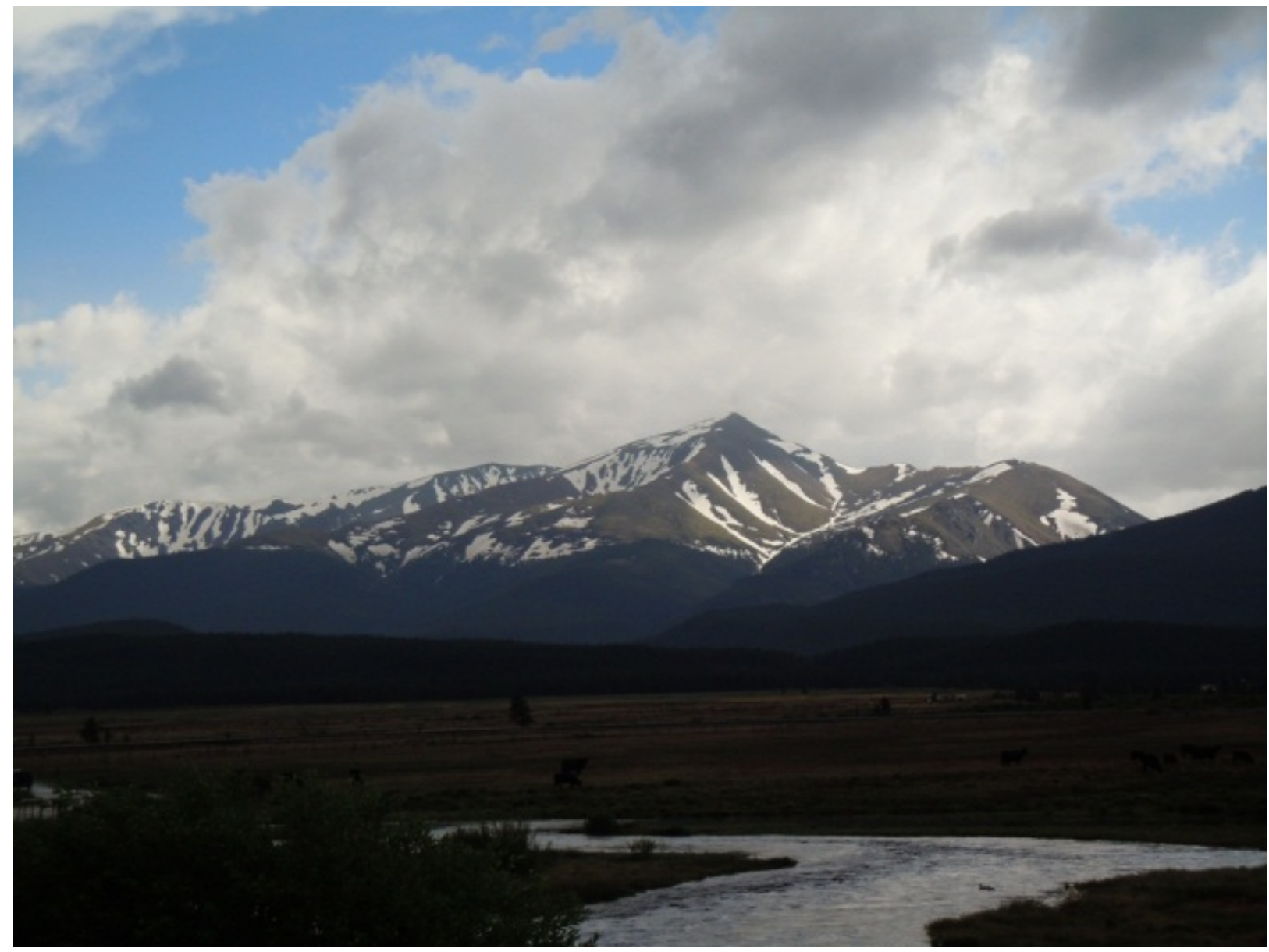

Open-File Report 2013-1064

U.S. Department of the Interior

U.S. Geological Survey 


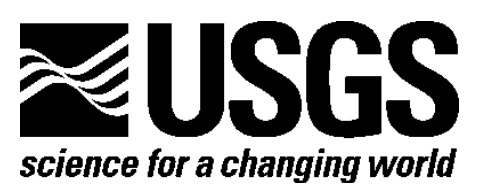

\section{Geochemical Results from Stream-Water and Stream- Sediment Samples Collected in Colorado and New Mexico}

By Philip L. Hageman, Andrew S. Todd, Kathleen S. Smith, Ed DeWitt, and Mathew P. Zeigler

Open-File Report 2013-1064

U.S. Department of the Interior

U.S. Geological Survey 


\section{U.S. Department of the Interior \\ SALLY JEWELL, Secretary}

\section{U.S. Geological Survey \\ Suzette M. Kimball, Acting Director}

U.S. Geological Survey, Reston, Virginia: 2013

For more information on the USGS-the Federal source for science about the Earth, it's natural and living resources, natural hazards, and the environment-visit http://www.usgs.gov or call 1-888-ASK-USGS

For an overview of USGS information products, including maps, imagery, and publications, visit http://www.usgs.gov/pubprod

To order this and other USGS information products, visit http://store.usgs.gov

\section{Suggested citation:}

Hageman, P.L., Todd, A.S., Smith, K.S., DeWitt, Ed, and Zeigler, M.P., 2013, Geochemical results from streamwater and stream-sediment samples collected in Colorado and New Mexico: U.S. Geological Survey Open-File Report 2013-1064, 11 p., http://pubs.usgs.gov/of/2013/1064/.

Any use of trade, product, or firm names is for descriptive purposes only and does not imply endorsement by the U.S. Government.

Although this report is in the public domain, permission must be secured from the individual copyright owners to reproduce any copyrighted material contained within this report.

Cover. View of Mount Elbert, Colorado. 


\section{Contents}

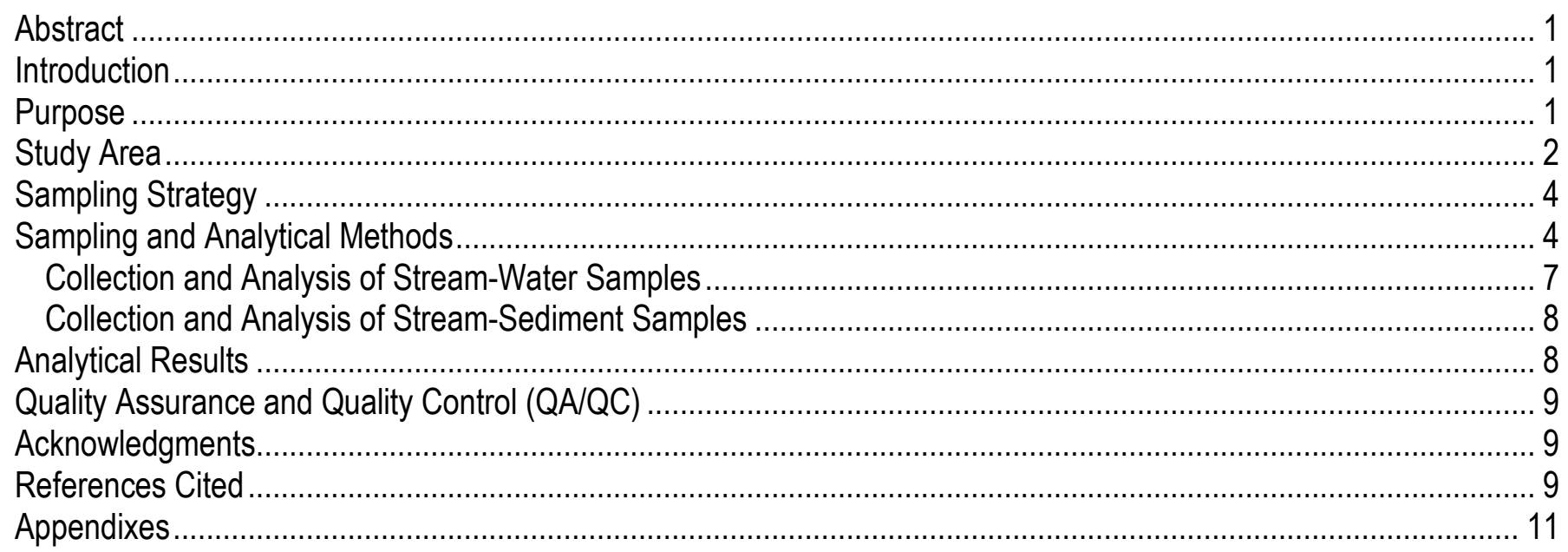

\section{Figure}

1. Distribution of sample sites included in this study

\section{Tables}

1. Details for stream-water samples and stream-sediment samples collected for this study .................................5

2. Details for collection of stream-water samples .................................................................................... 7

\section{Appendixes}

1. Bulk chemistry for 42 elements (ICP-AES), forms of carbon, and total sulfur for stream-sediment samples collected for this study...... link

2. Composition of filtered, acidified (FA) stream-water samples ...................................................................... link

3. Composition of unfiltered, acidified (RA) stream-water samples ................................................................ link

4. Quality assurance/quality control results for stream-sediment samples including bulk chemistry for 40 elements (ICP-AES), forms of carbon, and total sulfur. link

5. Quality assurance/quality control results for filtered-acidified (FA) stream-water samples ................................ link

6. Quality assurance/quality control results for unfiltered-acidified (RA) stream-water samples............................. link 


\title{
Geochemical Results from Stream-Water and Stream- Sediment Samples Collected in Colorado and New Mexico
}

\author{
By Philip L. Hageman, Andrew S. Todd, Kathleen S. Smith, Ed DeWitt, and Mathew P. Zeigler
}

\begin{abstract}
Scientists from the U.S. Geological Survey are studying the relationship between watershed lithology and stream-water chemistry. As part of this effort, 60 stream-water samples and 43 corresponding stream-sediment samples were collected in 2010 and 2011 from locations in Colorado and New Mexico. Sample sites were selected from small to midsize watersheds composed of a high percentage of one rock type or geologic unit. Stream-water and stream-sediment samples were collected, processed, preserved, and analyzed in a consistent manner. This report releases geochemical data for this phase of the study.
\end{abstract}

\section{Introduction}

In a natural setting, the geochemical characteristics of stream water are determined by the terrain through which the water flows (Miller, 2001). Further, it is understood that the mineralogic composition of the rocks in a watershed is a fundamental factor in determining the chemical makeup of the water emanating from such a watershed (Miller, 1999). However, the specific characteristics of water chemistry produced by interaction of stream water with particular rock types are not well defined. Understanding the rock/water relationship is important because water dissolves and incorporates the soluble components of the rocks, minerals, soil, and biota it comes into contact with both overland and as groundwater. Subsequent changes to stream-water or groundwater chemistry as a result of these interactions can influence, modify, or control important stream-water characteristics such as $\mathrm{pH}$, specific conductance, and the concentration of dissolved constituents. Fluctuation or changes in one or more of these key parameters may result in significant impacts to the viability or health of biota in an ecosystem. Because of the significance of these potential effects, it would be useful to be able to predict lithologically induced changes to stream-water chemistry.

\section{Purpose}

The purpose of this study is to collect and analyze stream-water and stream-sediment samples from watersheds that are dominated by distinct or specific rock types to determine if unique relationships are expressed between rock type and stream-water chemistry. This publication releases geochemical data from this study to other project scientists and to the public. Data included in this report consist of sample site coordinates, geologic detail for major rock types, and analytical results for stream-water and stream-sediment samples. The data are released without interpretation. Please note that data for water temperature and baseflow discharge for many of the streams included in this study can be found in Zeigler and others (2013). 


\section{Study Area}

Samples were collected from a variety of watersheds in the central and southern Rocky Mountains in Colorado and New Mexico (fig. 1). The study area encompasses a large, geologically diverse region, and because of its large size, the climate, vegetation, and topography of the sampling sites vary widely. In general, the summertime climate in the study area ranges from cool and humid in the high mountain areas to semiarid to arid at lower elevations. The natural vegetation in the area is diverse and strongly zoned according to elevation. It ranges from tree-free, alpine areas above the timberline to spruce, fir, and pine forests with some aspen and grassy meadows at middle to high elevations. Ponderosa, pinyon pine, and juniper dominate sites at lower elevations, with the lowest and most arid sampling sites dominated by a variety of shrubs, grasses, cacti, and yucca. 


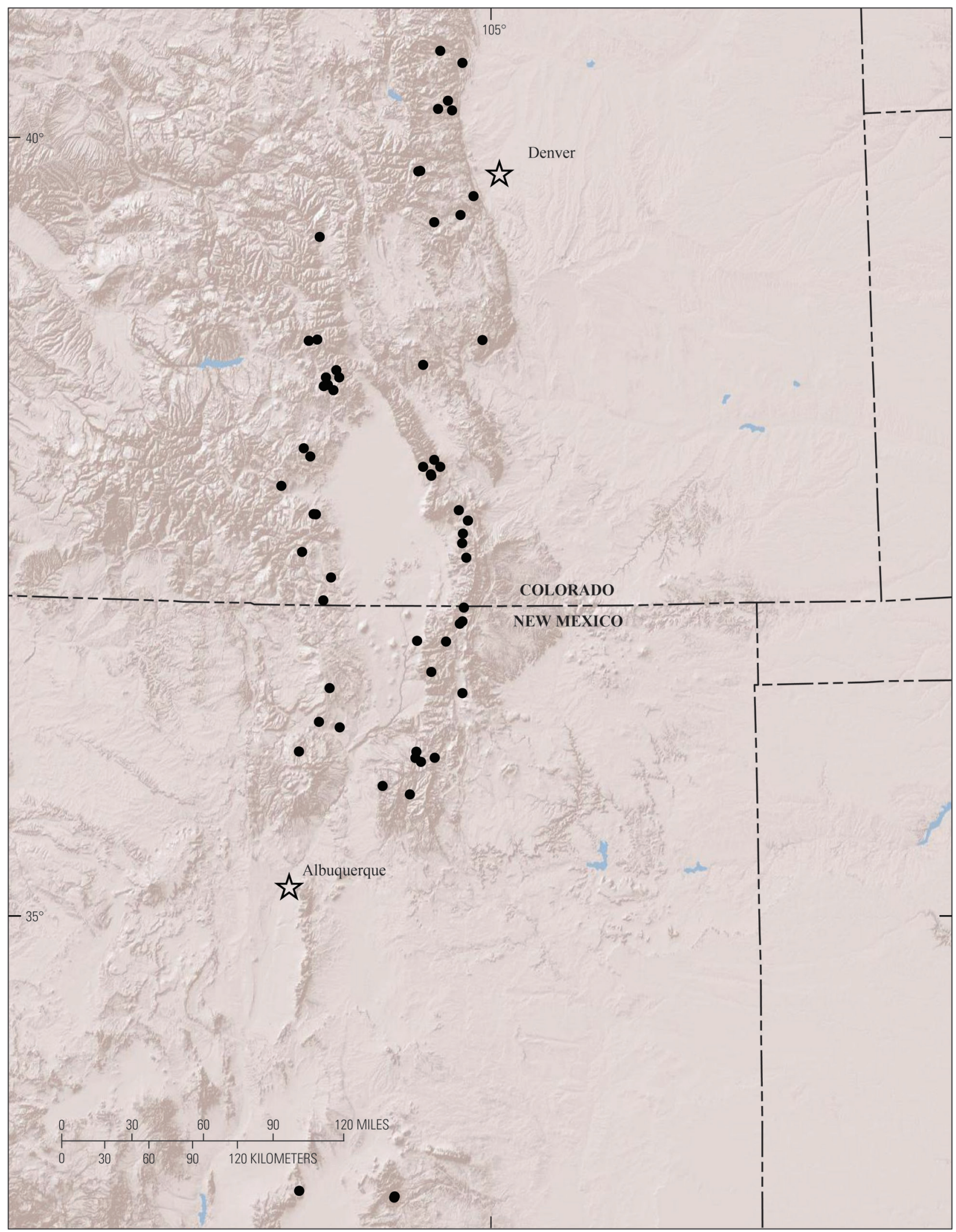

Figure 1. Distribution of sample sites included in this study. 


\section{Sampling Strategy}

A team of U.S. Geological Survey (USGS) personnel used geologic maps and geographic information systems (GIS) to select sampling sites that were determined to be in watersheds dominated by one rock type, lithology, or geologic unit. Selected sites were plotted on hard-copy topographic maps $(1: 24,000)$, and the coordinates were entered into a commercial, hand-held global positioning system (GPS) unit. The sampling plan was to collect both a stream-water sample and corresponding streamsediment sample from each location. However, because of onsite conditions, stream-sediment samples were not available at all sampling locations, so not all stream-water samples have corresponding streamsediment samples. In addition, two stream-sediment samples do not have corresponding stream-water samples because of dry conditions at those sampling sites. Most samples were collected during base flow in the late summer or early fall of 2010 and 2011.

\section{Sampling and Analytical Methods}

Stream-water samples were collected from small to midsize streams at 60 sites in Colorado and New Mexico. Corresponding stream-sediment samples were collected from a total of 43 sites as noted in table 1 . 
Table 1. Sample details for stream-water and stream-sediment samples collected for this study.

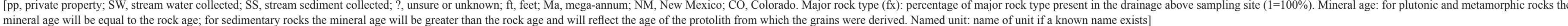

\begin{tabular}{|c|c|c|c|c|c|c|c|c|c|c|c|c|c|}
\hline Sample & State & Latitude & Longitude & Elevation (ft.) & sw & ss & Major rock type(s) & $\begin{array}{c}\text { Major rock } \\
\text { type (fx) }\end{array}$ & $\begin{array}{l}\text { Mineral age } \\
\text { (Ma) }\end{array}$ & Formation age & $\begin{array}{l}\text { Rock type* } \\
\text { (chemical) }\end{array}$ & Named unit & Stream or site \\
\hline ALA & NM & 36.055 & -105.468 & 9,608 & $\mathrm{x}$ & $\mathrm{x}$ & mixed clastic & 1 & 1,600 & Permian-Pennsylvanian & psammite & Sandia? & Alamitos Creek \\
\hline Aud & $\mathrm{CO}$ & 40.090 & -105.596 & 10,771 & $\mathrm{x}$ & $\mathrm{x}$ & syenite & 1 & 68 & Cretaceous & syenite & Audubon & Audubon \\
\hline CAB-L & NM & 36.736 & -105.508 & 8,537 & $\mathrm{X}$ & $\mathrm{x}$ & andesite, felsic, and aphantic volcanic rocks & 0.2 & 20 & Tertiary-Proterozoic mix & dacite mix & unnamed & Cabresto, lower site \\
\hline CAB-U & NM & 36.772 & -105.386 & 10,129 & $\mathrm{x}$ & & andesite, felsic volcanic rock & 0.2 & 20 & Tertiary & dacite mix & unknown & Cabresto, upper site \\
\hline $\mathrm{CaC}$ & $\mathrm{CO}$ & 37.908 & -106.449 & 9,085 & $\mathrm{X}$ & $\mathrm{x}$ & andesite & 1 & 20 & Tertiary & andesite & unknown & Cave Creek \\
\hline CAN-U & NM & 36.087 & -106.502 & 8,360 & $\mathrm{x}$ & $\mathrm{x}$ & felsic volcanic rocks, rhyolite & 1 & 20 & Tertiary & rhyodacite & Jemez & Canones Creek, upper site \\
\hline CC-A & $\mathrm{CO}$ & 39.876 & -105.300 & 7,176 & $\mathrm{x}$ & & quartzite & 1 & 1,700 & Proterozoic & quartzite & Coal Creek & Coal Creek-A \\
\hline $\cos$ & NM & $\mathrm{pp}$ & $\mathrm{pp}$ & 10,250 & $\mathrm{x}$ & $\mathrm{x}$ & basalt, clastic & 0.7 & 20 & Tertiary & basalt-sedimentary mix & Costilla Creek? & Costilla Creek \\
\hline DOR & $\mathrm{CO}$ & 38.396 & -106.336 & 9,291 & $\mathrm{x}$ & $\mathrm{x}$ & dolomite, sandstone & 1 & 440 & Devonian-Ordovician & dolomite-sandstone & Parting-Manitou & Indian Creek \\
\hline ELR & NM & 36.481 & -106.273 & 8,399 & $\mathrm{x}$ & $\mathrm{x}$ & mixed clastic & 0.8 & 1,200 & Tertiary & psammite & unknown & El Rito Creek \\
\hline EMC & $\mathrm{CO}$ & 38.320 & -106.276 & 9,957 & $\mathrm{x}$ & $\mathrm{x}$ & andesite, ash-flow tuff & 1 & 20 & Tertiary & andesite & unknown & East Middle Creek \\
\hline ESB & NM & 36.030 & -105.574 & 10,335 & $\mathrm{X}$ & $\mathrm{x}$ & mixed clastic & 1 & 1,600 & Permian-Pennsylvanian & psammite & Sandia? & Santa Barbara Creek, east fork \\
\hline Fair-A & $\mathrm{CO}$ & 38.621 & -106.475 & 9,696 & $\mathrm{X}$ & $\mathrm{x}$ & granodiorite & 1 & 1,690 & Proterozoic & granodiorite & Fairview Peak & Fairview-A \\
\hline FRJ & NM & 36.271 & -105.408 & 9,620 & $\mathrm{x}$ & & clastic & 1 & 1,600 & Permian-Pennsylvanian & psammite & Sandia? & Frijoles Creek \\
\hline HM-1 & $\mathrm{CO}$ & 38.636 & -105.107 & 8,732 & $\mathrm{X}$ & & granite & 1 & 1,700 & Proterozoic & granite & Brind Mountain & Henry Mountain-1 \\
\hline $\mathrm{HM}-2$ & $\mathrm{CO}$ & 38.636 & -105.107 & 9,175 & $\mathrm{X}$ & & granite & 1 & 1,700 & Proterozoic & granite & Henry Mountain & Henry Mountain-2 \\
\hline IND & NM & 36.091 & -105.608 & 8,914 & $\mathrm{X}$ & $\mathrm{x}$ & sandstone & 1 & 1,600 & Permian-Pennsylvanian & psammite & Sandia? & Indian Creek \\
\hline ITA & NM & 36.585 & -105.497 & 8,723 & $\mathrm{X}$ & $\mathrm{x}$ & plutonic rock (phaneritic) & 0.2 & 1,500 & Proterozoic-Tertiary mix & mixture & unnamed & Itallianos Creek \\
\hline JAC-L & NM & 35.828 & -105.657 & 8,302 & $\mathrm{x}$ & $\mathrm{x}$ & mixed clastic & 1 & 1,600 & Permian-Pennsylvanian & psammite & Sandia? & Jacks Creek, lower site \\
\hline JAC-U & NM & 35.875 & -105.648 & 9,985 & $\mathrm{X}$ & $\mathrm{x}$ & mixed clastic & 1 & 1,600 & Permian-Pennsylvanian & psammite & Sandia? & Jacks Creek, upper site \\
\hline JSA & $\mathrm{CO}$ & 40.111 & -105.387 & 6,998 & $\mathrm{X}$ & & quartz monzonite & 1 & 74 & Cretaceous & monzodiorite & Jamestown & Jamestown Stock, site A \\
\hline Kno-A & $\mathrm{CO}$ & 39.144 & -106.457 & 10,778 & $\mathrm{x}$ & & granodiorite & 1 & 1,690 & Proterozoic & granodiorite & Kroenke & Kroenke-A \\
\hline LFC & $\mathrm{CO}$ & 37.318 & -106.501 & 9,664 & $\mathrm{x}$ & $\mathrm{x}$ & andesite & 0.9 & 25 & Tertiary & andesite & unknown & Conejos, Lake Fork \\
\hline LM-1 & $\mathrm{CO}$ & 37.804 & -105.512 & 8,550 & $\mathrm{x}$ & & gneiss & 0.9 & 1,700 & Proterozoic & meta-psammite & unnamed & Little Medano Creek \\
\hline LMC & $\mathrm{CO}$ & 37.796 & -105.504 & 8,453 & $\mathrm{X}$ & & felsic gneiss, conglomerate, granite & 0.5 & 1,600 & Proterozoic mix & meta-psammite & unnamed & Medano Creek, lower site \\
\hline LTR & NM & 36.044 & -105.672 & 8,984 & $\mathrm{x}$ & $\mathrm{x}$ & meta-sedimentary, plutonic rocks & 0.8 & 1,690 & Proterozoic & meta-psammite & unnamed & Rio de las Trampas \\
\hline MCCRY & NM & 36.778 & -105.099 & 8,008 & $\mathrm{x}$ & & sandstone, landslide deposits and colluvium, piedmont alluvial deposits & 0.8 & $900 ?$ & Tertiary? & siltstone & $?$ & McCrystal \\
\hline McI & $\mathrm{CO}$ & 38.573 & -105.588 & 8,970 & $\mathrm{x}$ & $\mathrm{x}$ & mix: metabasalt, metadacite, metagabbro & 1 & 1,700 & Proterozoic & diorite-metabasalt & unnamed & McIntyre Gulch \\
\hline ME-1 & $\mathrm{CO}$ & 39.676 & -105.609 & 9,585 & $\mathrm{x}$ & & granodiorite-diorite & 1 & 1,440 & Proterozoic & granodiorite & Mount Evans & Chicago Creek \\
\hline ML-A & $\mathrm{CO}$ & 38.740 & -106.431 & 11,007 & $\mathrm{x}$ & & metabasalt, meta-andesite, minor granodiorite & 1 & 1,750 & Proterozoic & metabasalt & Ohio & Mirror Lake-A \\
\hline Mon-A & $\mathrm{CO}$ & 39.602 & -105.822 & 10,840 & $\mathrm{x}$ & $\mathrm{x}$ & granodiorite & 1 & 40 & Tertiary & granodiorite & Montezuma & Montezuma-A \\
\hline Mpr & $\mathrm{CO}$ & 38.629 & -106.406 & 10,800 & $\mathrm{x}$ & $\mathrm{x}$ & granodiorite-tonalite & 1 & 37 & Tertiary & granodiorite & Mount Princeton & Mount Princeton \\
\hline MRR & NM & $\mathrm{pp}$ & pp & 7,535 & $\mathrm{X}$ & $\mathrm{x}$ & felsic volcanic rocks, plutonic rock (phaneritic) & 0.5 & 40 & Tertiary & syenite & Sierra Blanca & Rio Ruidoso \\
\hline MSB & NM & 36.053 & -105.615 & 9,348 & $\mathrm{x}$ & $\mathrm{x}$ & mixed clastic & 1 & 1,600 & Permian-Pennsylvanian & psammite & Sandia? & Rio Santa Barbara, middle fork \\
\hline NRR & NM & $\mathrm{pp}$ & $\mathrm{pp}$ & 7,595 & $\mathrm{x}$ & $\mathrm{x}$ & granite, andesite & 0.6 & 40 & Tertiary & syenite & Sierra Blanca & Rio Ruidoso, north fork \\
\hline NTR & $\mathrm{CO}$ & $\mathrm{pp}$ & $\mathrm{pp}$ & 8,791 & $\mathrm{x}$ & $\mathrm{x}$ & felsic gneiss & 1 & 1,730 & Proterozoic & granodiorite & $\begin{array}{l}\text { Trinchera? } \\
\text { The }\end{array}$ & North Trinchera Creek \\
\hline $\mathrm{OC}$ & $\mathrm{CO}$ & 38.397 & -106.230 & 10,625 & $\mathrm{x}$ & $\mathrm{x}$ & metabasalt, meta-andesite, metadacite & 0.9 & 1,700 & Proterozoic & meta-andesite & unnamed & Ouray Creek \\
\hline OSI & $\mathrm{CO}$ & 37.020 & -106.333 & 9,776 & $\mathrm{x}$ & $\mathrm{x}$ & ash-flow tuff, mixed clastic (volcanic), landslide deposits & $0.6 ?$ & 25 & Tertiary & quartz latite & unknown & Osier Creek \\
\hline PC & $\mathrm{CO}$ & 37.957 & -106.500 & 9,857 & $\mathrm{x}$ & $\mathrm{x}$ & andesite & 1 & 25 & Tertiary & andesite & unnamed & Prong Creek \\
\hline Pee-D & $\mathrm{CO}$ & 39.584 & -106.996 & 7,051 & & $\mathrm{x}$ & evaporite & 1 & 300 & Permian-Pennsylvanian & evaporite & Eagle Valley & Eagle Valley evaporite \\
\hline Pet-B & $\mathrm{CO}$ & 40.577 & -105.461 & 8,258 & $\mathrm{x}$ & $\mathrm{x}$ & metagraywacke & $1 ?$ & 1,700 & Proterozoic & metawacke & unnamed & Petite-B \\
\hline PLAC & $\mathrm{CO}$ & pp & $\mathrm{pp}$ & 8,569 & $\mathrm{x}$ & $\mathrm{x}$ & felsic gneiss, sandstone, plutonic rock (phaneritic) & 0.6 & 1,500 ? & Proterozoic mix? & granite? & North Russell? & Placer Creek \\
\hline Pmb-A & $\mathrm{CO}$ & 39.441 & -106.672 & 9,816 & $\mathrm{x}$ & $\mathrm{x}$ & black shale, sandstone & 0.8 & 300 & Pennsylvanian? & C-rich pelite & Belden? & Crooked Creek \\
\hline PNL & NM & 33.626 & -105.245 & 6,317 & $\mathrm{x}$ & $\mathrm{x}$ & plutonic, sedimentary & 1 & 40 & Tertiary & syenite & El Capitan & $\begin{array}{l}\text { Pinelodge } \\
\text { Pan }\end{array}$ \\
\hline PPww & $\mathrm{CO}$ & 39.616 & -106.996 & 7,420 & & $\mathrm{x}$ & sandstone & 0.9 & 1,500 & Permian-Pennsylvanian & psammite & Minturn? & Red beds \\
\hline Raw & $\mathrm{CO}$ & 40.701 & -105.761 & 7,820 & $\mathrm{x}$ & $\mathrm{x}$ & granite & 1 & 1,720 & Proterozoic & granite & Rawah & Rawah \\
\hline Roos-A & $\mathrm{CO}$ & 38.584 & -106.539 & 9,088 & $\mathrm{x}$ & & granite & 1 & 1,760 & Proterozoic & alkali granite & Roosevelt & Roosevelt-A \\
\hline $\mathrm{SC}$ & $\mathrm{CO}$ & 37.851 & -105.571 & 8,299 & $\mathrm{X}$ & & granite, felsic gneiss, conglomerate & 0.6 & 1,500 & Proterozoic mix & granite-gneiss & Music Mountain? & Sand Creek \\
\hline SC-1 & $\mathrm{CO}$ & 39.675 & -105.622 & 9,627 & $\mathrm{x}$ & & mix: granodiorite, metagraywacke & 1 & 1,500 & Proterozoic & granodiorite-metawacke mix & Mount Evans--unnamed & South Chicago Creek \\
\hline SF-U & $\mathrm{CO}$ & 37.551 & -106.398 & 9,751 & $\mathrm{x}$ & $\mathrm{x}$ & andesite, ash-flow tuff & 0.8 & 25 & Tertiary & andesite & San Juan--name? & San Francisco Creek, upper site \\
\hline SPA & $\mathrm{CO}$ & 39.681 & -105.477 & 9,749 & $\mathrm{x}$ & & mix: metagraywacke, mafic metavolcanic rocks & 1 & 1,700 & Proterozoic & metawacke-metabasalt & unnamed & Squaw Pass-A \\
\hline SS-C & $\mathrm{CO}$ & 40.060 & -105.469 & 8,209 & $\mathrm{x}$ & & syenite & 1 & 55 & Tertiary & syenite & Sunset & Sunset Stock-C \\
\hline TRIUP & $\mathrm{CO}$ & $\mathrm{pp}$ & $\mathrm{pp}$ & 10,260 & $\mathrm{x}$ & $\mathrm{x}$ & felsic gneiss, siltstone, glacial drift & 0.5 & 1,500 & Proterozoic-Pennsylvanian mix & granite mix & unknown & Trinchera, upper site \\
\hline
\end{tabular}


Table 1. Sample details for stream-water and stream-sediment samples collected for this study-Continued

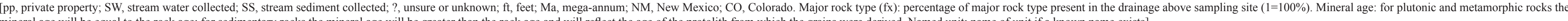
mineral age will be equal to the rock age; for sedimentary rocks the mineral age will be greater than the rock age and will reflect the age of the protolith from which the grains were derived. Named unit: name of unit if a known name exists

\begin{tabular}{|c|c|c|c|c|c|c|c|c|c|c|c|c|c|}
\hline Sample & State & Latitude & Longitude & Elevation (ft.) & sw & ss & Major rock type(s) & $\begin{array}{c}\text { Major rock } \\
\text { type }(f x)\end{array}$ & $\begin{array}{c}\text { Mineral age } \\
(\mathrm{Ma})\end{array}$ & Formation age & $\begin{array}{l}\text { Rock type* } \\
\text { (chemical) }\end{array}$ & Named unit & Stream or site \\
\hline TWO-L & NM & 36.898 & -105.261 & 9,436 & $\mathrm{X}$ & $\mathrm{x}$ & plutonic rocks, clastic & 0.4 & 1,700 & Proterozoic-Tertiary & granite mix & granite--name? & Casius Creek, lower site \\
\hline TWO-U & NM & 36.884 & -105.279 & 9,455 & $\mathrm{X}$ & $\mathrm{x}$ & plutonic rocks, clastic & 0.5 & 1,700 & Proterozoic-Tertiary & granite mix & granite--name? & Casius Creek, upper site \\
\hline UMC & $\mathrm{CO}$ & 37.852 & -105.438 & 9,650 & $\mathrm{x}$ & & conglomerate, felsic gneiss & 0.7 & 1,500 & Permian-Pennsylvanian & sandstone mix & Sangre de Cristo mix & Medano Creek, upper site \\
\hline USC & $\mathrm{CO}$ & 37.889 & -105.502 & 9,706 & $\mathrm{x}$ & $\mathrm{x}$ & granite, conglomerate, arkose & 0.7 & 1,400 & Proterozoic & granite & Music Mountain & Sand Creek, upper site \\
\hline WCR & $\mathrm{CO}$ & $\mathrm{pp}$ & $\mathrm{pp}$ & 8,674 & $\mathrm{x}$ & $\mathrm{x}$ & conglomerate, sandstone & 0.8 & 1,500 & Permian-Pennsylvanian & conglomerate & Madera? & Wagon Creek \\
\hline $\mathrm{WCr}$ & $\mathrm{CO}$ & 37.018 & -106.461 & 8,650 & $\mathrm{X}$ & $\mathrm{x}$ & andesite, unconsolidated & 0.9 & 25 & Tertiary & andesite & unknown & Wolf Creek \\
\hline WFA & $\mathrm{CO}$ & 37.723 & -106.670 & 8,700 & $\mathrm{x}$ & & volcanics & 0.9 & 25 & Tertiary & dacite? & unknown & Alder Creek, west fork \\
\hline WHA & $\mathrm{CO}$ & 37.925 & -106.698 & 10,312 & $\mathrm{X}$ & $\mathrm{x}$ & volcanics & 1 & 25 & Tertiary & latite & unknown & Whale Creek \\
\hline WIC & $\mathrm{CO}$ & $\mathrm{pp}$ & $\mathrm{pp}$ & 8,559 & $\mathrm{X}$ & $\mathrm{x}$ & sandstone, felsic gneiss, conglomerate & 0.4 & 1,500 & Proterozoic-Pennsylvanian mix & granite mix & unknown & West Indian Creek \\
\hline
\end{tabular}

"Igneous rocks classified using De la Roche and others (1980), metasedimentary rocks classified by Ed DeWitt (U.S Geological Survey, unpub.data, 2012). 


\section{Collection and Analysis of Stream-Water Samples}

At each sampling location, coordinates and site details were logged and a stream-water sample was collected midstream using a pre-cleaned 500-milliliter $(\mathrm{mL})$ high-density polyethylene (HDPE) plastic bottle that was rinsed three times with site water. Immediately following collection of the streamwater sample, $\mathrm{pH}$ and specific conductance (SC) were determined on unfiltered subsamples using calibrated, hand-held meters. Next, the stream-water samples were filtered and preserved using the procedures listed in table 2 .

\section{Table 2. Details for collection of stream-water samples.}

[FU, filtered, unacidified; FA, filtered, acidified; RA, unfiltered, acidified; Hg, mercury; Fe, iron; DOC, dissolved organic carbon; $\mathrm{cm}^{3}$, cubic centimeter; $\mu \mathrm{m}$, micrometer; $\mathrm{mL}$, milliliter; HDPE, high-density polyethylene; ${ }^{\circ} \mathrm{C}$, degree Celsius; $<$, less than]

\begin{tabular}{|c|c|c|c|c|}
\hline Sample split & Analyte(s) & Filtration details & Bottle type & Preservation \\
\hline FU & $\begin{array}{l}\text { Chloride, } \\
\text { fluoride, nitrate, } \\
\text { and alkalinity }\end{array}$ & $\begin{array}{l}60-\mathrm{cm}^{3} \text { syringe and a } \\
0.45-\mu \mathrm{m} \text { nylon capsule } \\
\text { filter }\end{array}$ & 60-mL HDPE & $\begin{array}{l}\text { Refrigeration at } 4^{\circ} \mathrm{C} \text { until } \\
\text { analyzed }\end{array}$ \\
\hline FA & $\begin{array}{l}\text { Cations and } \\
\text { sulfate }\end{array}$ & $\begin{array}{l}60-\mathrm{cm}^{3} \text { syringe and a } 0.45- \\
\mu \mathrm{m} \text { nylon capsule filter }\end{array}$ & $\begin{array}{l}\text { 30-mL acid- } \\
\text { washed HDPE }\end{array}$ & $\begin{array}{l}\text { Acidify to } \mathrm{pH}<1.7 \text { with } \\
\text { ultra-pure nitric acid ( } 2 \text { or } \\
3 \text { drops } / 30 \mathrm{~mL})\end{array}$ \\
\hline RA & $\begin{array}{l}\text { Cations and } \\
\text { sulfate }\end{array}$ & $\begin{array}{l}60-\mathrm{cm}^{3} \text { syringe, no } \\
\text { filtration }\end{array}$ & $\begin{array}{l}\text { 30-mL acid- } \\
\text { washed HDPE }\end{array}$ & $\begin{array}{l}\text { Acidify to } \mathrm{pH}<1.7 \text { with } \\
\text { ultra-pure nitric acid ( } 2 \text { or } \\
3 \text { drops } / 30 \mathrm{~mL} \text { ) }\end{array}$ \\
\hline $\mathrm{Hg}$ & Mercury & $\begin{array}{l}60-\mathrm{cm}^{3} \text { syringe and a } 0.45- \\
\mu \mathrm{m} \text { nylon capsule filter }\end{array}$ & $\begin{array}{l}\text { 30-mL acid- } \\
\text { washed flint glass } \\
\text { bottle with Teflon- } \\
\text { lined lid }\end{array}$ & $\begin{array}{l}\text { Acidify with ultra-pure } \\
\text { hydrochloric acid (1 } \\
\mathrm{mL} / 30 \mathrm{~mL})\end{array}$ \\
\hline $\mathrm{Fe}^{2+}$ & Ferrous iron & $\begin{array}{l}60-\mathrm{cm}^{3} \text { syringe and a } 0.45- \\
\mu \mathrm{m} \text { nylon capsule filter }\end{array}$ & $\begin{array}{l}60-\mathrm{mL} \text { amber } \\
\text { HDPE }\end{array}$ & $\begin{array}{l}\text { Acidify with ultra-pure } \\
\text { concentrated hydrochloric } \\
\text { acid ( } 3 \text { drops } / 60 \mathrm{~mL})\end{array}$ \\
\hline $\mathrm{DOC}$ & $\begin{array}{l}\text { Dissolved organic } \\
\text { carbon }\end{array}$ & $\begin{array}{l}60-\mathrm{cm}^{3} \text { syringe and a } 0.45- \\
\mu \mathrm{m} \text { polyethersulfone } \\
(\mathrm{PES}) \text { capsule filter }\end{array}$ & $\begin{array}{l}125-\mathrm{mL} \text { pre-baked } \\
\text { amber glass bottles }\end{array}$ & $\begin{array}{l}\text { Acidify with ultra-pure } \\
\text { hydrochloric acid ( } 5 \\
\text { drops } / 125 \mathrm{~mL})\end{array}$ \\
\hline
\end{tabular}

Upon return from the field, all water samples were submitted to the USGS Central Region Mineral and Environmental Science Center (CRMESC) laboratories in Lakewood, Colorado, for log-in and analysis. Pertinent sample information was entered into the Laboratory Information Management System (LIMS), and each sample was given a unique laboratory identification number. The samples were then distributed to the laboratories for chemical analyses using the following methods:

- FU (filtered, unacidified) samples were analyzed for chloride $\left(\mathrm{Cl}^{-}\right)$, fluoride $\left(\mathrm{F}^{-}\right)$, and nitrate $\left(\mathrm{NO}_{3}\right)$ using ion chromatography (IC) (Theodorakos and others, 2002); a separate split of this sample was analyzed for alkalinity by titration (Theodorakos, 2002a).

- Both the FA (filtered, acidified) and RA (unfiltered, acidified) samples were submitted for cation and sulfate analysis by inductively coupled plasma-atomic emission spectrometry (ICP-AES) 
(Briggs, 2002a) and inductively coupled plasma-mass spectrometry (ICP-MS) (Lamothe and others, 2002).

- Ferrous iron $\left(\mathrm{Fe}^{2+}\right)$ was determined by spectrophotometry (Theodorakos, 2002b).

- Dissolved mercury (Hg) was determined using cold vapor-atomic fluorescence (CVAFS) (Hageman, 2007).

- Dissolved organic carbon (DOC) was determined by combustion-infrared detection (Shimadzu Corporation, 1997).

\section{Collection and Analysis of Stream-Sediment Samples}

For this study, stream-sediment samples were collected (when present) from active streambed alluvium within 10 meters $(\mathrm{m})$ of the plotted sample locality. The samples were composited by taking increments of material from several streambed locations using a plastic scoop. If limited stream sediment was available, a single bulk sediment sample was collected from bed-load material. Sample size for most sites was approximately 1 kilogram $(\mathrm{kg})$. All stream-sediment samples were wet-sieved onsite using a 10-mesh (2-millimeter [mm]) stainless steel screen and a plastic pan. The material that passed the screen was taken directly from the pan and saved for processing and analysis.

Upon return from the field, the sediment samples were logged into the Laboratory Information System (LIMS) at the USGS Central Region Mineral and Environmental Science Center (CRMESC), and each sample was given a unique laboratory identification number. The samples were then prepared using the following procedure. First, the sediments were air-dried at ambient temperature. Dried samples were then mixed and split using a Jones splitter. One split of the bulk material was returned to the submitter for archive, and the other split was processed for laboratory analysis by dry sieving to -80 mesh $(0.17 \mathrm{~mm})$. The portion of the sediment that passed the sieve was then pulverized using a shatter box to approximately -150 mesh $(0.1 \mathrm{~mm})$. Splits of ground material were then placed into pre-labeled cardboard containers that were sealed and sent to the USGS contract laboratories for determination of 40 elements using a four acid digestion and analysis by ICP-AES (Briggs, 2002b). The contract laboratory also determined forms of carbon (C) using the following methods:

- Total $\mathrm{C}$ was determined by combustion using an automated carbon analyzer (Brown and Curry, 2002).

- Carbonate $\mathrm{C}$ was determined using coulometric titration of $\mathrm{CO}_{2}$ evolved after treatment of the sample with hot $2 \mathrm{~N}$ perchloric acid (Brown and others, 2002).

- Organic $\mathrm{C}$ was calculated from the difference between total $\mathrm{C}$ and carbonate $\mathrm{C}$.

The contract laboratory also determined total sulfur using an automated sulfur analyzer.

\section{Analytical Results}

Bulk-chemistry results for the stream-sediment samples are found in appendix 1. Analytical results for all filtered acidified (FA) stream-water samples are provided in appendix 2. Results for unfiltered acidified (RA) samples are found in appendix 3. Please note that analytical data for zinc ( $\mathrm{Zn}$ ) for the stream-water samples were not included in the dataset because of a contamination problem. Bulk zinc, however, is reported for the stream-sediment samples. Samples in all the tables are organized alphabetically according to their sample identification for ease of cross-reference. 


\section{Quality Assurance and Quality Control (QA/QC)}

For stream sediments, site duplicate samples were collected at three of the sampling locations. These samples were collected concurrently with the primary sample using all the same equipment, processes, and procedures. Duplicate samples were submitted blind as part of the sample set. In addition, prior to submittal to the contract laboratories, blind reference samples (Standard L, Standard $\mathrm{M}$, and Standard Ras-A) were integrated into the sample sets by the submitter. Additional reference standards were inserted into the sample set by USGS Sample Control. These internal reference samples were submitted with the sample set at a ratio of 10 percent and were run through the analytical procedures as blind samples. Analytical data for the internal reference samples are assessed as part of the USGS quality control system. Quality assurance and quality control (QA/QC) data for Standard L, Standard M, Standard Ras-A, and the duplicate samples are found in appendix 4.

Stream-water samples for QA/QC include both field and laboratory procedure blanks, site duplicate samples, and a blind reference sample (T-167). These samples were submitted to the laboratories as part of the analytical dataset. The field blank was processed in-situ using the same procedures and equipment used for collection of the stream-water samples. The water used for the field blank sample was deionized water (NanoPure, $18 \mathrm{M} \Omega$ ) from the laboratory and was carried into the field in a clean 500-mL HDPE bottle. The laboratory blanks were processed and prepared with all the procedures and equipment used for collection of the stream-water samples with the exception that they were prepared in the laboratory. Water used for the laboratory blanks was deionized water (NanoPure, $18 \mathrm{M} \Omega$ ). Stream-water duplicate samples were collected concurrently with the primary sample using the same equipment and procedures as those used to collect all the stream-water samples. In addition, all of the analytical techniques used for this study employ extensive QA/QC protocols, which are described in an online publication (Taggart, 2002). The quality control data for the stream-water FA and RA samples are provided in appendix 5 and appendix 6, respectively.

\section{Acknowledgments}

We thank the following individuals who assisted us with GIS, sample collection, or sample analysis: John Horton, Rene Galindo, Cyrus Berry, Monique Adams, Mike Anthony, and David Fey. Funding for this study was provided by the USGS Mineral Resources Program.

\section{References Cited}

Briggs, P.H., 2002a, The determination of twenty-seven elements in aqueous samples by inductively coupled plasma-atomic emission spectrometry, chap. F of Taggart, J.E., Jr., ed., Analytical methods for chemical analysis of geologic and other materials: U.S. Geological Survey Open-File Report 02223, p. F1-F11.

Briggs, P.H., 2002b, The determination of forty elements in geological and botanical samples by inductively coupled plasma-atomic emission spectrometry, chap. G of Taggart, J.E., Jr., ed., Analytical methods for chemical analysis of geologic and other materials: U.S. Geological Survey Open-File Report 02-223, p. G1-G20.

Brown, Z.A., and Curry, K.J., 2002, Total carbon by combustion, chap. R of Taggart, J.E., Jr., ed., Analytical methods for chemical analysis of geologic and other materials: U.S. Geological Survey Open-File Report 02-223, p. R1-R4. 
Brown, Z.A., Papp, Clara, Brandt, Elaine, and Aruscavage, Phillip, 2002, Carbonate carbon by coulometric titration, chap. S of Taggart, J.E., Jr., ed., Analytical methods for chemical analysis of geologic and other materials: U.S. Geological Survey Open-File Report 02-223, p. S1-S6.

De la Roche, H., Leterrier, J., Grandclaude, P., and Marchal, M., 1980, A classification of volcanic and plutonic rocks using $\mathrm{R}_{1}, \mathrm{R}_{2}$-diagrams and major element analysis-Its relationships with current nomenclature: Chemical Geology, v. 29, p. 183-210.

Hageman, P.L., 2007, Determination of mercury in aqueous and geologic materials by continuous flow- $\square$ cold vapor-atomic fluorescence spectrometry (CVAFS): U.S. Geological Survey Techniques and Methods, book 5, chap. D2, 6 p.

Lamothe, P.J., Meier, A.L., and Wilson, S.A., 2002, The determination of forty-four elements in aqueous samples in inductively coupled plasma-mass spectrometry, chap. H of Taggart, J.E., Jr., ed., Analytical methods for chemical analysis of geologic and other materials: U.S. Geological Survey Open-File Report 02-223, p. H1-H11.

Miller, W.R., 1999, Geochemical baselines and maps showing acid-neutralizing capacity and potential release of total dissolved solids of stream and spring waters from different rock compositional types from mountainous watersheds in the Gunnison, Uncompahgre, and Grand Mesa National Forest, Colorado: U.S. Geological Survey Open-File Report 99-580, 107 p.

Miller, W.R., 2001, Geochemical baselines of stream and spring waters from areas underlain by Permian and Pennsylvanian rocks, including evaporites, and potential environmental damage in the Eagle Valley, Colorado: U.S. Geological Survey Open-File Report 01-362, 41 p.

Shimadzu Corporation, 1997, Model TOC 5000-An instrument manual: Kyoto, Japan, Shimadzu Corporation, $155 \mathrm{p}$.

Taggart, J.E., ed., 2002, Analytical methods for chemical analysis of geologic and other materials, U.S. Geological Survey: U.S. Geological Survey Open-File Report 02-223, variously paged. (Also available at http://pubs.usgs.gov/of/2002/ofr-02-0223/.)

Theodorakos, P.M., 2002a, Determination of total alkalinity using a preset endpoint ( $\mathrm{pH} 4.5)$ auto titration system, chap. E of Taggart, J.E., Jr., ed., Analytical methods for chemical analysis of geologic and other materials: U.S. Geological Survey Open-File Report 02-223, p. E1-E3.

Theodorakos, P.M., 2002b, Colorimetric determination of ferrous iron, Fe(II), in natural water, wastewater, and seawater, chap. W of Taggart, J.E., Jr., ed., Analytical methods for chemical analysis of geologic and other materials: U.S. Geological Survey Open-File Report 02-223, p. W1-W2.

Theodorakos, P.M., d'Angelo, W.M., and Ficklin, W.H., 2002, Fluoride, chloride, nitrate, and sulfate in aqueous solution utilizing AutoSupression chemically suppressed ion chromatography, chap. V of Taggart, J.E., Jr., ed., Analytical methods for chemical analysis of geologic and other materials: U.S. Geological Survey Open-File Report 02-223, p. V1-V7.

Zeigler, M.P., Todd, A.S., and Caldwell, C.A., 2013, Water temperature and baseflow discharge of streams throughout the range of Rio Grande cutthroat trout in Colorado and New Mexico-2010 and 2011: U.S. Geological Survey Open-File Report 2013-1051, 18 p., http://pubs.usgs.gov/of/2013/1051/. 


\section{Appendixes}

1. Bulk chemistry for 42 elements (ICP-AES), forms of carbon, and total sulfur for streamsediment samples collected for this study

2. Composition of filtered, acidified (FA) stream-water samples

3. Composition of unfiltered, acidified (RA) stream-water samples

4. Quality assurance/quality control results for stream-sediment samples including bulk chemistry for 40 elements (ICP-AES), forms of carbon, and total sulfur

5. Quality assurance/quality control results for filtered, acidified (FA) stream-water samples

6. Quality assurance/quality control results for unfiltered, acidified (RA) stream-water samples 